\title{
An one-pot three-component process for the synthesis of 6-amino-4-aryl-5-cyano-3-methyl-1-phenyl-1,4- dihydropyrano[2,3-c]pyrazoles in aqueous media
}

\author{
Tong-Shou Jin*, Rui-Qiao Zhao, and Tong-Shuang Li \\ College of Chemistry and Environmental Science, Hebei University, \\ Baoding 071002, P. R. China \\ E-mail: jintongshou@yahoo.com.cn
}

\begin{abstract}
A three-component process for the one-pot synthesis of 6-amino-4-aryl-5-cyano-3-methyl-1phenyl-1,4-dihydropyrano[2,3-c]pyrazoles from 3-methyl-1-phenyl-2-pyrazolin-5-one, aromatic aldehydes and malononitrile using $p$-dodecylbenzenesulfonic acid (DBSA) as the catalyst (10 mol \%) in aqueous media is described. This method provides several advantages such as mild reaction conditions, simple work-up procedure and is environment friendly. In addition, water is chosen as a green solvent.
\end{abstract}

Keywords: Pyrano[2,3-c]pyrazole, 3-methyl-1-phenyl-2-pyrazolin-5-one, aromatic aldehyde, malononitrile, clean synthesis

\section{Introduction}

The $21^{\text {th }}$ century is the modern year of the green chemistry, more and more chemists are devoted to the research of the 'green synthesis' which means the reagent, solvent and catalyst are environmental friendly. Recently organic reactions in water without use of harmful organic solvents have attracted much attention, because water is a cheap, safe and environmentally benign solvent. ${ }^{1}$ However, water as a solvent was not frequently used until recently for several reasons such as many organic materials do not dissolve in water and many reactive intermediates and catalysts are decomposed in water. So it is necessary to add some phase-transfer catalyst (PTC) or surfactant such as hexadecyltrimethylammonium bromide (HTMAB), tetrabutylammonium bromide (TBAB), p-dodecylbenesulfonic acid (DBSA), because they benefit the organic materials uniform dispersion in water in the course of synthesis.

It is known that polyfunctionalized benzopyrans and their derivatives are a kind of very useful compounds. They have been widely used as medicine intermediates due to their useful biological and pharmacological properties, such as antibacterial, anticoagulant, anticancer, 
spasmolytic, hypnotic, diuretic, insecticide. ${ }^{2-6}$ Some 2-amino-4H-pyrans can be employed as photoactive meterials. ${ }^{7}$ Furthermore, multisubstitutional $4 H$-pyrans also constitute a structural unit of a series of natural products. ${ }^{8,9}$ Besides, substituted pyrazole and derivatives can be used as important pharmaceuticals and agricultural chemicals. Usually these compounds are synthesized in organic solvents. ${ }^{10,11}$ In the course of our investigations to develop new synthetic methods in water. We have completed a series of organic syntheses with water as solvent recently. ${ }^{12}$ Herein we report a one-pot three-component and highly efficient method for synthesis of 6-amino-4aryl-5-cyano-3- methyl-1-phenyl-1,4-dihydropyrano[2,3-c]pyrazoles catalyzed by DBSA in aqueous media. This is an efficient synthesis in aqueous media, not only the operational simplicity but also gives the corresponding products in good to excellent yields. (Scheme 1).

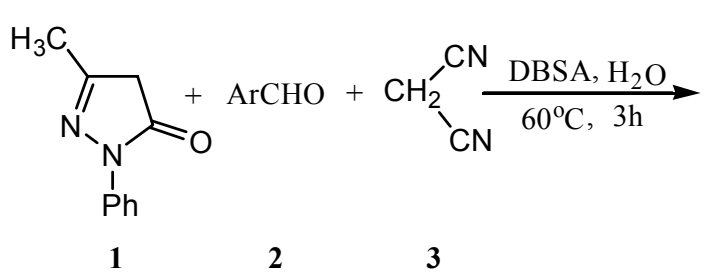

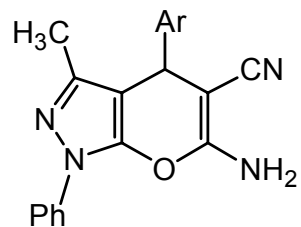

4

Scheme 1

In the presence of DBSA, 3-methyl-1-phenyl-2-pyrazolin-5-one 1, aromatic aldehyde 2 and malononitrile 3 were performed in water at $60{ }^{\circ} \mathrm{C}$, high yields of products 4 were obtained. The results are summarized in Table 1.

Table 1. Synthesis of pyrano[2,3-c]pyrazoles catalysed by DBSA in aqueous media

\begin{tabular}{|c|l|c|c|c|}
\hline Entry & Ar & Yield (\%) & \multicolumn{2}{|c|}{ Mp $\left.{ }^{\mathbf{0}} \mathbf{C}\right)$} \\
\hline & & & Found & Reported \\
\hline 1 & $\mathrm{C}_{6} \mathrm{H}_{5} 4 \mathrm{a}$ & 88 & $170-171$ & $168-170$ \\
\hline 2 & $4-\mathrm{CH}_{3} \mathrm{C}_{6} \mathrm{H}_{4} 4 \mathrm{~b}$ & 87 & $177-178$ & $176-178$ \\
\hline 3 & $4-\mathrm{CH}_{3} \mathrm{OC}_{6} \mathrm{H}_{4} 4 \mathrm{c}$ & 86 & $171-172$ & $170-172$ \\
\hline 4 & $3,4-\left(\mathrm{OCH}_{2} \mathrm{O}\right) \mathrm{C}_{6} \mathrm{H}_{3} 4 \mathrm{~d}$ & 82 & $175-176$ & $174-176$ \\
\hline 5 & $4-\mathrm{HOC}_{6} \mathrm{H}_{4} 4 \mathrm{e}$ & 84 & $210-212$ & $211-212$ \\
\hline 6 & $2-\mathrm{ClC}_{6} \mathrm{H}_{4} 4 \mathrm{f}$ & 92 & $145-146$ & $144-146$ \\
\hline 7 & $3-\mathrm{ClC}_{6} \mathrm{H}_{4} 4 \mathrm{~g}$ & 90 & $158-160$ & $158-159$ \\
\hline 8 & $4-\mathrm{ClC}_{6} \mathrm{H}_{4} 4 \mathrm{~h}$ & 92 & $175-176$ & $174-175$ \\
\hline 9 & $2,4-\mathrm{Cl}_{2} \mathrm{C}_{6} \mathrm{H}_{3} 4 \mathrm{i}$ & 94 & $185-186$ & $182-184$ \\
\hline 10 & $3-\mathrm{NO}_{2} \mathrm{C}_{6} \mathrm{H}_{4} 4 \mathrm{j}$ & 90 & $190-191$ & $188-190$ \\
\hline 11 & $4-\mathrm{NO}_{2} \mathrm{C}_{6} \mathrm{H}_{4} 4 \mathrm{k}$ & 92 & $195-196$ & $194-196$ \\
\hline 12 & $4-\left(\mathrm{CH}_{3}\right)_{2} \mathrm{NC}_{6} \mathrm{H}_{4} 41$ & no reaction & & \\
\hline
\end{tabular}

${ }^{a}$ Isolated yield. 
As shown in Table 1, we can find a series of aromatic aldehyde $\mathbf{2}$ were reacted with $\mathbf{1}$ and 3 in the presence of DBSA in aqueous media at $60{ }^{\circ} \mathrm{C}$, the reaction proceeds smoothly to afford the corresponding 6-amino-4-aryl-5-cyano-3-methyl-1-phenyl-1,4-dihydropyrano[2,3c]pyrazoles 4 in good to excellent yields. No very obvious effect of the electronic nature of substituents in the aromatic ring was observed. Benzaldehyde and aromatic aldehydes containing electron-donating groups (such as alkyl group, alkoxyl group, hydroxyl group) or electronwithdrawing groups (such as halide, nitro group) were employed and reacted well to give the corresponding products 4 in good to excellent yields under this reaction conditions.

The catalyst (DBSA) plays a crucial role in the success of the reaction in terms of the rate and the yields. For example, the reaction of 2,4-dichlorobenzaldehyde, $\mathbf{1}$ and $\mathbf{3}$ could be carried out in the absence of DBSA when the mixture $(\mathbf{1}, \mathbf{2 i}$ and $\mathbf{3})$ in aqueous media at $60{ }^{\circ} \mathrm{C}$ for $6 \mathrm{~h}$, but the yield is poor (24\%). Additionally, 4-dimethylaminobenzaldehyde (11) failed to give the corresponding 6-amino-4-aryl-5-cyano-3-methyl-1-phenyl-1,4-dihydropyrano[2,3-c]pyrazole and the starting materials were quantitatively recovered under the same conditions. The explanation for this result may be due to the strong electron donating dimethylamino group in (11) which will reduce the reactivity. A degree of tautomerisation may occur in (11) with formation of quinoid structure and thus decreased reactivity of the aldehyde group (Scheme 2).

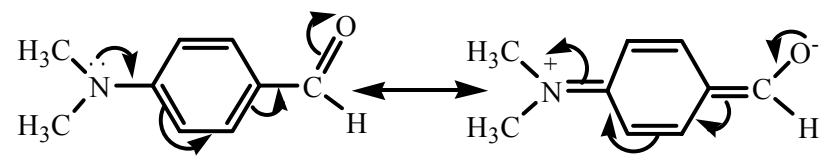

\section{Scheme 2}

We propose the possible following mechanism to account for the reaction. One molecule of aromatic aldehyde $\mathbf{2}$ was firstly condensed with malononitrile $\mathbf{3}$ to afford $\alpha$ cyanocinnamonitrile derivative $\mathbf{5}$. The step $(\mathbf{2}+\mathbf{3} \rightarrow \mathbf{5})$ can be regarded as a fast Knoevenagel addition. The active methylene of $\mathbf{1}$ by reaction with the electrophilic $\mathrm{C}=\mathrm{C}$ double bond gave the intermediate 6 tautomerization to the intermediate 7. Then the intermediate 7 cyclised by the nucleophilic attack of $\mathrm{OH}$ group on the cyano $(\mathrm{CN})$ moiety and gave the intermediate $\mathbf{8}$, finally the expected products 4 tautomerised from $8(7 \rightarrow \mathbf{8} \rightarrow \mathbf{4})$. In this process, DBSA could promote these reactions as an emulsifier (Scheme 3). 


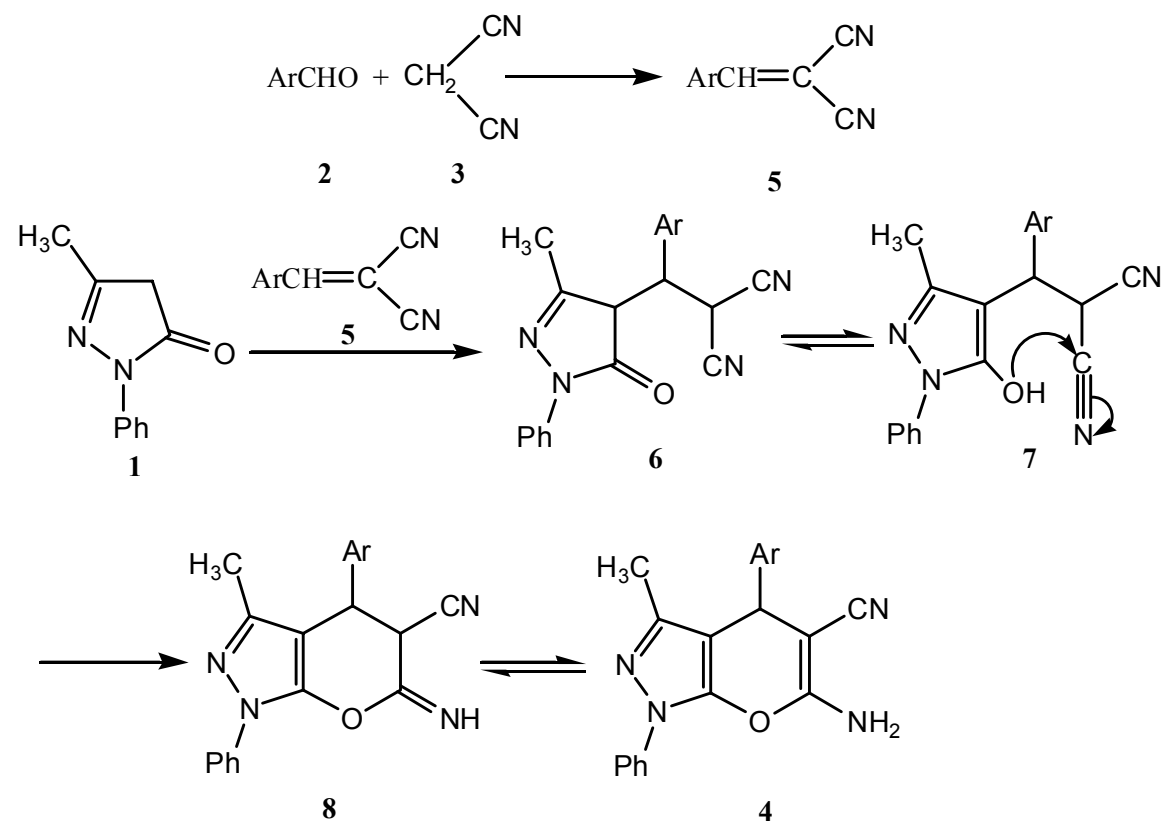

\section{Scheme 3}

In conclusion, a procedure for the preparation of 6-amino-4-aryl-5-cyano-3-methyl-1phenyl-1,4-dihydropyrano[2,3-c]pyrazoles catalyzed by DBSA in aqueous media have been developed. This is a one-pot three-component condensation in aqueous media. Water solution is a clean and environmentally desirable system. No harmful organic solvents are used. This report has proposed and demonstrated a new useful and attractive process for the synthesis of these compounds.

\section{Experimental Section}

General Procedures. Liquid aldehydes were distilled before use. IR spectra were recorded on a Bio-Rad FTS-40 spectrometer (KBr). ${ }^{1} \mathrm{H}$ NMR spectra were measured on a Bruker AVANCE $400(400 \mathrm{MHz})$ spectrometer using TMS as internal reference and $\mathrm{CDCl}_{3}$ as solvent. Elemental analyses were determined using Perkin-Elmer 2400 II elemental analyzer.

General procedure for the preparation of 4. A mixture of 3-methyl-1-phenyl-2-pyrazolin-5one $(1,2 \mathrm{mmol})$, aromatic aldehyde $(2,2 \mathrm{mmol})$, malononitrile $(3,2 \mathrm{mmol})$, and DBSA (10 mol\%) in water $(40 \mathrm{~mL})$ was stirred at $60{ }^{\circ} \mathrm{C}$ for 3 hours. Then the mixture was cooled to room temperature, solid was filtered off and washed with $\mathrm{H}_{2} \mathrm{O}(40 \mathrm{~mL})$. The crude products were purified by recrystallization by ethanol $(95 \%)$ to give 4 . Data of compounds are shown below:

6-Amino-5-cyano-3-methyl-1,4-diphenyl-1,4-dihydropyrano[2,3-c]pyrazole (4a). IR ( $\mathrm{KBr):}$ $v_{\max }=3472,3320,2195,1660,1590,1264,1125,1027,753 \mathrm{~cm}^{-1} .{ }^{1} \mathrm{H}$ NMR: $\delta=1.93(\mathrm{~s}, 3 \mathrm{H}$, $\left.\mathrm{CH}_{3}\right), 4.68(\mathrm{~s}, 1 \mathrm{H}, 4-\mathrm{H}), 4.75\left(\mathrm{~s}, 2 \mathrm{H}, \mathrm{NH}_{2}\right), 7.16-7.32(\mathrm{~m}, 10 \mathrm{H}, \mathrm{ArH}) \mathrm{ppm}$. Anal. Calcd. for $\mathrm{C}_{20} \mathrm{H}_{16} \mathrm{~N}_{4} \mathrm{O}$ : C 73.15, H 4.91, N 17.06; found C 73.19, H 5.01, N 17.10. 
6-Amino-4-(4-methylphenyl)-5-cyano-3-methyl-1-phenyl-1,4-dihydropyrano[2,3-c]pyrazole (4b). IR (KBr): $v_{\max }=3414,3314,2178,1658,1594,1398,1258,1128,1026,754 \mathrm{~cm}^{-1} .{ }^{1} \mathrm{H}$ NMR: $\delta=1.78\left(\mathrm{~s}, 3 \mathrm{H}, \mathrm{CH}_{3}\right), 2.28\left(\mathrm{~s}, 3 \mathrm{H}, \mathrm{CH}_{3}\right), 4.62(\mathrm{~s}, 1 \mathrm{H}, 4-\mathrm{H}), 6.96\left(\mathrm{~s}, 2 \mathrm{H}, \mathrm{NH}_{2}\right), 7.02(\mathrm{~d}, 2 \mathrm{H}$, $J=8.4 \mathrm{~Hz}, \mathrm{ArH}), 7.08$ (d, 2H, $J=8.4 \mathrm{~Hz}, \mathrm{ArH}), 7.30-7.34$ (m, 1H, ArH), 7.46 (d, 2H, J=8.0 Hz, $\operatorname{ArH}), 7.78(\mathrm{~d}, 2 \mathrm{H}, J=8.0 \mathrm{~Hz}, \mathrm{ArH}) \mathrm{ppm}$. Anal. Calcd. for $\mathrm{C}_{21} \mathrm{H}_{18} \mathrm{~N}_{4} \mathrm{O}$ : C 73.67, H 5.30, N 16.36; found C 73.71, H 5.34, N 16.42 .

6-Amino-4-(4-methoxyphenyl)-5-cyano-3-methyl-1-phenyl-1,4-dihydropyrano[2,3-c] pyrazole (4c). IR (KBr): $v_{\max }=3395,3322,2192,1660,1595,1394,1250,1128,813 \mathrm{~cm}^{-1} .{ }^{1} \mathrm{H}$ NMR: $\delta=1.78\left(\mathrm{~s}, 3 \mathrm{H}, \mathrm{CH}_{3}\right), 3.72\left(\mathrm{~s}, 3 \mathrm{H}, \mathrm{OCH}_{3}\right), 4.88(\mathrm{~s}, 1 \mathrm{H}, 4-\mathrm{H}), 6.82(\mathrm{~d}, 2 \mathrm{H}, J=8.0 \mathrm{~Hz}, \operatorname{ArH})$, 6.96 (s, 2H, NH$), 7.04$ (d, 2H, J=8.0 Hz, ArH), 7.20-7.24 (m, 1H, ArH), 7.40 (d, 2H, J=8.0 Hz, ArH), 7.58 (d, $2 \mathrm{H}, J=8.0 \mathrm{~Hz}, \mathrm{ArH})$ ppm. Anal. Calcd. for $\mathrm{C}_{21} \mathrm{H}_{18} \mathrm{~N}_{4} \mathrm{O}_{2}$ : C 70.38, H 5.06, N 15.63; found C 70.42, H 5.11, N 15.68 .

6-Amino-4-(3,4-dioxymethylenephenyl)-5-cyano-3-methyl-1-phenyl-1,4-dihydropyrano[2,3-c]pyrazole (4d). IR (KBr): $v_{\max }=3400,3320,2198,1661,1595,1393,1254,1129,1037$, $789 \mathrm{~cm}^{-1} .{ }^{1} \mathrm{H}$ NMR: $\delta=2.12\left(\mathrm{~s}, 3 \mathrm{H}, \mathrm{CH}_{3}\right), 5.10(\mathrm{~s}, 1 \mathrm{H}, 4-\mathrm{H}), 5.94\left(\mathrm{~s}, 2 \mathrm{H}, \mathrm{OCH}_{2} \mathrm{O}\right), 6.93(\mathrm{~s}, 2 \mathrm{H}$, $\left.\mathrm{NH}_{2}\right), 7.02-7.12(\mathrm{~m}, 2 \mathrm{H}, \mathrm{ArH}), 7.14$ (m, 1H, ArH), 7.18 (d, 2H, J=8.0 Hz, ArH), 7.20 (m, 1H, ArH), 7.26 (d, 2H, J=8.0 Hz, ArH) ppm. Anal. Calcd. for $\mathrm{C}_{21} \mathrm{H}_{16} \mathrm{~N}_{4} \mathrm{O}_{3}: \mathrm{C}$ 67.73, H 4.33, N 15.05; found C 67.78, H 4.38, N 15.11 .

6-Amino-4-(4-hydroxyphenyl)-5-cyano-3-methyl-1-phenyl-1,4-dihydropyrano[2,3-c]pyrazole (4e). IR (KBr): $v_{\max }=3414,3314,2178,1658,1594,1398,1258,1128,1026,754 \mathrm{~cm}^{-1} .{ }^{1} \mathrm{H}$ NMR: $\delta=1.78\left(\mathrm{~s}, 3 \mathrm{H}, \mathrm{CH}_{3}\right), 4.56(\mathrm{~s}, 1 \mathrm{H}, 4-\mathrm{H}), 6.72(\mathrm{~d}, 2 \mathrm{H}, J=8.4 \mathrm{~Hz}, \mathrm{ArH}), 7.04(\mathrm{~d}, 2 \mathrm{H}, J=8.4$ $\mathrm{Hz}, \mathrm{ArH}), 7.12$ (s, 2H, NH ), 7.30-7.34 (m, 1H, ArH), 7.48 (d, 2H, J=8.0 Hz, ArH), 7.78 (d, 2H, $J=8.0 \mathrm{~Hz}, \mathrm{ArH}$ ) ppm. Anal. Calcd. for $\mathrm{C}_{20} \mathrm{H}_{16} \mathrm{~N}_{4} \mathrm{O}_{2}$ : C 69.76, H 4.68, N 16.27; found C 69.82, $\mathrm{H}$ $4.72, \mathrm{~N} 16.31$.

6-Amino-4-(2-chlorophenyl)-5-cyano-3-methyl-1-phenyl-1,4-dihydropyrano[2,3-c]pyrazole (4f). IR (KBr): $v_{\max }=3472,3324,2194,1656,1592,1389,1264,1125,1028,752 \mathrm{~cm}^{-1} .{ }^{1} \mathrm{H}$ NMR: $\delta=1.90\left(\mathrm{~s}, 3 \mathrm{H}, \mathrm{CH}_{3}\right), 4.72\left(\mathrm{~s}, 2 \mathrm{H}, \mathrm{NH}_{2}\right), 5.32(\mathrm{~s}, 1 \mathrm{H}, 4-\mathrm{H}), 7.22-7.44(\mathrm{~m}, 5 \mathrm{H}, \mathrm{ArH}), 7.48$ (d, $2 \mathrm{H}, J=8.0 \mathrm{~Hz}, \mathrm{ArH}), 7.68(\mathrm{~d}, 2 \mathrm{H}, J=8.0 \mathrm{~Hz}, \mathrm{ArH}) \mathrm{ppm}$. Anal. Calcd. for $\mathrm{C}_{20} \mathrm{H}_{15} \mathrm{ClN}_{4} \mathrm{O}: \mathrm{C}$ 66.21, H 4.17, N 15.44; found C 66.26, H 4.22, N 15.48.

6-Amino-4-(3-chlorophenyl)-5-cyano-3-methyl-1-phenyl-1,4-dihydropyrano[2,3-c]pyrazole (4g). IR (KBr): $v_{\max }=3462,3318,2194,1656,1594,1392,1070,756 \mathrm{~cm}^{-1} .{ }^{1} \mathrm{H}$ NMR: $\delta=1.92$ $\left(\mathrm{s}, 3 \mathrm{H}, \mathrm{CH}_{3}\right), 4.68(\mathrm{~s}, 1 \mathrm{H}, 4-\mathrm{H}), 4.74\left(\mathrm{~s}, 2 \mathrm{H}, \mathrm{NH}_{2}\right), 7.18-7.38(\mathrm{~m}, 5 \mathrm{H}, \mathrm{ArH}), 7.48$ (d, 2H, J=8.0 $\mathrm{Hz}, \mathrm{ArH}), 7.68(\mathrm{~d}, 2 \mathrm{H}, J=8.0 \mathrm{~Hz}, \mathrm{ArH}) \mathrm{ppm}$. Anal. Calcd. for $\mathrm{C}_{20} \mathrm{H}_{15} \mathrm{ClN}_{4} \mathrm{O}: \mathrm{C}$ 66.21, H 4.17, N 15.44; found C 66.25, H 4.21, N 15.50 .

6-Amino-4-(4-chlorophenyl)-5-cyano-3-methyl-1-phenyl-1,4-dihydropyrano[2,3-c]pyrazole (4h). IR (KBr): $v_{\max }=3468,3325,2200,1662,1596,1390,1262,1122,1016,752 \mathrm{~cm}^{-1} .{ }^{1} \mathrm{H}$ NMR: $\delta=1.88\left(\mathrm{~s}, 3 \mathrm{H}, \mathrm{CH}_{3}\right), 4.74\left(\mathrm{~s}, 2 \mathrm{H}, \mathrm{NH}_{2}\right), 6.32(\mathrm{~s}, 1 \mathrm{H}, 4-\mathrm{H}), 7.28-7.38(\mathrm{~m}, 3 \mathrm{H}, \mathrm{ArH}), 7.42$ (d, 2H, J=8.0 Hz, ArH), 7.48-7.52 (m, 2H, ArH), 7.78 (d, 2H, J=8.0 Hz, ArH) ppm. Anal. Calcd. for $\mathrm{C}_{20} \mathrm{H}_{15} \mathrm{ClN}_{4} \mathrm{O}$ : C 66.21, H 4.17, N 15.44; found C 66.26, H 4.19, N 15.52.

6-Amino-4-(2,4-dichlorophenyl)-5-cyano-3-methyl-1-phenyl-1,4-dihydropyrano[2,3-c]py- 
razole (4i). IR (KBr): $v_{\max }=3456,3322,2198,1660,1592,1392,1270,1126,1050,758 \mathrm{~cm}^{-1}$. ${ }^{1} \mathrm{H}$ NMR: $\delta=1.78\left(\mathrm{~s}, 3 \mathrm{H}, \mathrm{CH}_{3}\right), 5.14(\mathrm{~s}, 1 \mathrm{H}, 4-\mathrm{H}), 6.74\left(\mathrm{~s}, 2 \mathrm{H}, \mathrm{NH}_{2}\right), 7.32-7.78(\mathrm{~m}, 8 \mathrm{H}, \mathrm{ArH})$ ppm. Anal. Calcd. for $\mathrm{C}_{20} \mathrm{H}_{14} \mathrm{Cl}_{2} \mathrm{~N}_{4} \mathrm{O}$ : C 60.47, H 3.55, N 14.10; found C 60.51, H 3.58, N 14.16. 6-Amino-4-(3-nitrophenyl)-5-cyano-3-methyl-1-phenyl-1,4-dihydropyrano[2,3-c]pyrazole (4j). IR (KBr): $v_{\max }=3420,3330,2194,1675,1598,1390,1264,1126,1030,752 \mathrm{~cm}^{-1} .{ }^{1} \mathrm{H}$ NMR: $\delta=1.94\left(\mathrm{~s}, 3 \mathrm{H}, \mathrm{CH}_{3}\right), 4.76(\mathrm{~s}, 1 \mathrm{H}, 4-\mathrm{H}), 4.98\left(\mathrm{~s}, 2 \mathrm{H}, \mathrm{NH}_{2}\right), 8.12-8.95(\mathrm{~m}, 9 \mathrm{H}, \mathrm{ArH}) \mathrm{ppm}$. Anal. Calcd. for $\mathrm{C}_{20} \mathrm{H}_{15} \mathrm{~N}_{5} \mathrm{O}_{3}$ : C 64.34, $\mathrm{H} 4.05, \mathrm{~N} 18.76$; found $\mathrm{C} 64.38 \mathrm{H} \mathrm{4.10,} \mathrm{N} \mathrm{18.81.}$ 6-Amino-4-(4-nitrophenyl)-5-cyano-3-methyl-1-phenyl-1,4-dihydropyrano[2,3-c]pyrazole (4k). IR (KBr): $v_{\max }=3430,3340,2192,1665,1596,1354,1124,832,754 \mathrm{~cm}^{-1}$. ${ }^{1} \mathrm{H}$ NMR: $\delta=1.80\left(\mathrm{~s}, 3 \mathrm{H}, \mathrm{CH}_{3}\right), 4.96(\mathrm{~s}, 1 \mathrm{H}, 4-\mathrm{H}), 6.98\left(\mathrm{~s}, 2 \mathrm{H}, \mathrm{NH}_{2}\right), 7.32-7.36(\mathrm{~m}, 1 \mathrm{H}, \mathrm{ArH}), 7.48-7.52(\mathrm{~m}$, 2H, ArH), 7.58 (d, 2H, J=8.4 Hz, ArH), 7.80 (d, 2H, J=8.4 Hz, ArH), 8.24 (d, 2H, ArH) ppm. Anal. Calcd. for $\mathrm{C}_{20} \mathrm{H}_{15} \mathrm{~N}_{5} \mathrm{O}_{3}$ : C 64.34, $\mathrm{H} 4.05, \mathrm{~N} 18.76$; found $\mathrm{C} 64.40 \mathrm{H} 4.08, \mathrm{~N} 18.80$.

\section{Acknowledgements}

This project was supported by the National Natural Science Foundation of China (29872011), Educational Ministry of China, Science and Technology Commission of Hebei Province.

\section{References}

1. Li, C. J.; Chan, T. H. Organic Reaction in Aqueous Media, Wiley: New York, 1997.

2. Foye, W. O. Prinicipi di Chimica Farmaceutica Piccin, Padova: Italy, 1991, p 416.

3. Adreani, L. L.; Lapi, E. Boll. Chim. Farm. 1960, 99, 583; Chem. Abstr. 1961, 55, 2668 d.

4. Zhang, Y. L.; Chen, B. Z.; Zheng, K. Q.; Xu, M. L.; Lei, X. H. Chinese Acta Pharmaceutica Sinica 1982, 17, 17; Chem. Abstr. 1982, 96, 135383e.

5. Bonsignore, L.; Loy, G..; Secci, D.; Calignano, A. Eur. J. Med. Chem. 1993, $28,517$.

6. (a) Witte, E. C.; Neubert, P.; Roesch, A. Ger. Offen DE. 1986, 3427985; Chem. Abstr. 1986, 104, 224915f. (b) Wang, J. L.; Liu, D.; Zhang, Z. J.; Shan, S.; Han, X.; Srinivasula, S. M.; Croce, C. M.; Alnemri, E. S.; Huang, Z. Proc. Natl. Acad. Sci. U. S. A. 2000, 97, 7124. (c) Monamed, Y. A.; Zahran, M. A.; Ali, M. M.; ElAgrody, A. M.; El-Said, U. H. J. Chem. Res (S). 1995, 322.

7. Armetso, D.; Horspool, W. M.; Martin, N.; Ramos, A.; Seaone, C. J. Org. Chem. 1989, 54, 3069.

8. Hatakeyama, S.; Ochi, N.; Numata; H.; Takano, S. J. Chem. Soc., Chem. Commun. 1988, 1202.

9. (a) Gonzalez, R.; Martin, N.; Seoane; C.; Soto, J. J. Chem. Soc., Perkin Trans. 1. 1985, 202.

(b) Kamaljit, S. J.; Harjit, S. Tetrahedron 1996, 52, 14273.

10. (a) Shi, D. Q.; Mou, J.; Zhuang, Q. Y.; Niu, L.H.; Wu, N.; Wang, X. S. Synth. Commun. 
2004, 34, 4557. (b) Zhou, J. F.; Tu, S. J.; Gao, Y.; Qi, M. Chinese J. Org. Chem. 2001, 21, 742.

11. Kamaljit, S.; Jasbi, S.; Harjit, S. Tetrahedron 1996, 52, 14273.

12. (a) Jin, T. S.; Zhang, J. S.; Xiao, J. C.; Wang, A.Q.; Li, T. S. Synlett 2004, 866. (b) Jin, T. S.; Wang, A.Q.; Wang, X.; Zhang, J. S.; Li, T. S. Synlett 2004, 871. (c) Jin, T. S.; Zhang, J. S.; Guo, T. T.; Wang, A.Q.; Li, T. S. Synthesis 2004, 2001. (d) Jin, T. S.; Wang, A.Q.; Cheng, Z. L.; Zhang, J. S.; Li, T. S. Synth. Commun. 2005, 35, 2339. (e) Jin, T. S.; Liu, L. B.; Zhao, Y.; Li, T. S. Synth. Commun. 2005, 35, 2379. (f) Jin, T. S.; Wang, A. Q.; Cheng, Z. L.; Zhang, J. S.; Li, T. S. Synth. Commun. 2005, 35,137. (g) Jin, T. S.; Liu, L. B.; Zhao, Y.; Li, T. S. Synth. Commun. 2005, 35, 1859. (h) Jin, T. S.; Zhang, J. S.; Wang, A.Q.; Zhang, F. S. Chinese J. Org. Chem. 2005, 25, 335. 